\title{
Strategies to Implement Big Data Analytics in Telecommunication Organizations
}

\author{
Dr. Delton Aneato \\ Walden University \\ Minneapolis MN, USA
}

\author{
Dr. Cesar Castellanos \\ Walden University \\ Minneapolis MN, USA
}

\begin{abstract}
Information technology (IT) leaders who do not invest in big data projects may struggle to gain a competitive advantage and business insights to improve performance. Grounded in Kotter's change and Six Sigma models, the purpose of this qualitative multiple case study was to explore strategies IT leaders use to implement big data analytics successfully. The participants comprised 4 IT leaders from 2 telecommunication organizations in the United States of America, who expertly used big data analytics strategies to promote and maximize competitive advantage. Data were collected from semistructured interviews, company documents, and projectrelated documents. The collected information was examined by utilizing a thematic analysis approach. Four themes emerged from the data analysis process communication, training, employee involvement in decisions, and teamwork strategy. A key recommendation from these findings is for IT leaders to use successful communication strategies to convey the vision and objectives to all organizational levels. The successful communication-strategy can help evaluate business trends, forecasts, improve overall organizational performance and competitive advantage. The implications for positive social change include the potential for job creation, thus catalysing economic growth within communities.
\end{abstract}

Keywords: Strategies to Implement Big Data; Big Data Analytics; Telecommunication Organizations; Managerial Approaches; Change Models

\section{INTRODUCTION}

Nearly half of organizational leaders invest in big data projects (Grover et al., 2018). Leaders who do not invest in big data projects may fail to recognize and leverage the information needed to gain a competitive advantage (Chrimes et al., 2017). Due to the notable cost reduction in data acquisition technology, data processing, and data storage, digital data's exponential growth to support business operations. Therefore, organizational leaders are forced to discover techniques to collect a vast amount of data, process the data, analyze and derive patterns that lead to business decisions. After attaining a desired systematic process, organizational leaders are to identify strategies to implement the right technology, process data successfully, and achieve the aspired outcome. Grover et al. indicated that big data adoption could have adverse effects after implementation. Grover et al. defended the argument by stating that big data implementation's low success rate can negatively affect operational and financial performance. This qualitative multiple case study aimed to explore the strategies used by IT leaders in the telecommunication industry and how big data analysis is used to drive decisions. Adopting big data analytics implementation strategies may help leaders successfully achieve business goals to improve organizational performance while reducing operating costs. Implementing big data analytics can improve a company's competitive advantage, growth, and performance. However, inefficiently applying big data analytics strategies can result in selecting the wrong technologies (Matthias et al., 2017). Big data technology enables organizational leaders to access, diagnose, and integrate the necessary information gathered through various data sources. Therefore, organizational leaders need to have the skills and techniques to collect and manage the data. Leaders also need to gain implementation experience and the knowledge to employ analytical tools. The success of implementing big data analytics requires that leaders have the expertise and knowledge of these strategies. Big data analytics drive organizational experience among leaders to improve their business goals. Alsghaier et al. (2017) advocated the view that big data analytics is related to effective operations, customer satisfaction, and positive financial performance. Potential benefits of using big data analytics within organizations exist, but organizations still fail to appreciate the value of such practice (Sharma et al., 2014). In large organizations, $50 \%$ of big data-related projects are never completed. However, organizational leaders still require substantial guidance to realize the value generated by using big data analytics (Wang \& Hajli, 2017). Organizational leaders should consider big data analytics seriously to heighten and evolve their development issues.

\section{PROBLEM \& PURPOSE OF THE STUDY}

Business leaders are not likely to benefit from using big data analytics if they do not recognize and leverage business patterns to improve processes or reduce costs (Chrimes et al., 2017). A 2016 survey indicated that $48 \%$ of organizational leaders invested in big data projects, but $60 \%$ of these organizational leaders struggled to gain a competitive advantage (Grover et al., 2018). The general business problem is that leaders lack strategies to use big data analytics to improve processes or reduce costs. The specific business problem is that some IT leaders in the telecommunication industry lack the knowledge to implement big data analytics successfully. The purpose of this qualitative multiple case study was to explore strategies that IT leaders in the telecommunication industry used to implement big data analytics successfully. The targeted population was comprised of four IT leaders in the telecommunication industry from two organizations that have successfully implement big data analytics. The geographic area for this research was Seattle, Washington, and New York, New York. Implications for positive social change include the potential improvement of the employees' standard of living by providing permanent well-paying jobs that enable employees to support their families and contribute to communities. 


\section{DISCUSSIONS}

\subsection{Background of Big Data Analytics}

Big data is defined as data that is continually being generated from multiple sources and diverse data formats that are structured and unstructured (Grover et al., 2018). Big data provides leaders the potential to mining valuable business information for their organization (Balachandran \& Prasad, 2017). Leaders can get a holistic view of their performance through the translation and consumption of structured and unstructured data from multiple sources. Big data can help leaders identify relationships, patterns, and insights from different types of data to help them determine their organization's performance. Wamba et al. (2015) defined big data analytics as the holistic approach of capturing, managing, processing, and analyzing data. A typical data classification method is the 5V's (i.e., volume, velocity, variety, value, and veracity).

Big data creates business insights for delivering sustainability, performance, value, and establishing competitive advantages over its competitors (Wamba et al., 2015). The first characteristic is volume, which refers to the ever-growing magnitude of data. Velocity refers to the continuous generation of data at an unparalleled pace. Variety is the different data formats, ranging from structured, semistructured, and unstructured data (Grover et al., 2018). Value refers to the worth of hidden insights in data. Finally, veracity refers to the biases, noise, trustworthiness, and messiness of the data set (Yaqoob et al., 2016). The emergence of networked businesses from social media outlets, including Facebook and Instagram, has dramatically increased the volume, variety, velocity, value, and veracity of structured, semistructured, and unstructured data. Traditional data analytic tools can process and store structured and semistructured data in conventional databases.

In contrast, big data analytics can store and process structured, semistructured, and unstructured data from multiple sources. Studying the origin of big data applications is vital in understanding the conceptual background, vision, and trend of big data (Yaqoob et al., 2016). Big data analytics has spawned into a massive industry of data. However, before its existence, standalone applications existed with a single processing unit to reflect business users' actions with the computation speed of a local host machine (Abolfazli et al., 2014). Moreover, leaders used these standalone machines (a PC or server) with no existing network but with software that stored the data, performed several calculations, and produced results for further analysis. The ability to run data analytics software locally on a standalone machine was a significant source of empowerment to leaders, and this led to an increase in the purchase of standalone corporate machines in the 1960s and 1970s, and then the era of personal computers (PCs) in the 1980s (Kacprzyk \& Zadrożny, 2001). Structured data has a high degree of order and use by organizational leaders, such that it can be stored in a relational database seamlessly, readily available for straightforward analysis and search engines (Adnan \& Akbar, 2019). Examples of structured data include numbers, groups of words, and dates stored in a relational database. Semistructured data does not conform to relational databases' formal structure (Woo et al., 2019). An example of semistructured data is XML which is used to transfer data from one destination to another. XML is a language used for data exchange and representation (Nassiri et al., 2018). Unstructured data cannot be stored in rows and columns in a relational database and has no identifiable structure (Wu \& Lin, 2018). Examples of unstructured data include archived documents in a file folder, e-mail messages, photos, videos, and images.

Three types of advanced analytical techniques exist in big data: (a) predictive analysis, which is responsible for developing models based on past data for future prediction; (b) descriptive analysis, which is a model that reports on the past; and (c) prescriptive analysis, which uses models to specify optimal actions and behaviors (Grover et al., 2018). Grover et al. mentioned that advanced analytical techniques provide a forward-looking view, enabling leaders to anticipate and execute future opportunities based on real-time insights discovered from current events, ongoing business processes, and high-volume streaming data sources. Technology is a crucial and complementary strategic asset to big data analytics. While much of the literature surrounding big data analytics is relatively new, big data analytics is a robust process by which leaders can unearth social and potentially immense economic value to gain a competitive advantage.

Technological advancements with the combination of advanced analytical techniques can enable organizational leaders to realize the benefits of using big data analytics (Balachandran \& Prasad, 2017). According to Grover et al. (2018), big data analytics apply statistical technology, processing, and advanced analytical techniques to big data to advance a competitive advantage. The International Data Corporation showed in 2019 that big data technology and services markets increased at a $23.1 \%$ compound annual rate, reaching \$48.6 billion. Several big data technologies exist in the market. Some include Hadoop and NoSQL technologies that provide leaders with access to real-time, centralized data collected from different sources. As Grover et al. indicated, leaders can embrace unique opportunities to capitalize on big data analytics, which can be translated to competitive advantage.

Big data provides leaders with the potential to mine useful business information for their organization (Balachandran \& Prasad, 2017). Leaders can get a holistic view of their performance through the translation and consumption of structured and unstructured data from multiple sources. Big data can help leaders identify relationships, patterns, and insights from different data types to help them determine their organization's performance. The use of big data by leaders can help improve organizational growth by using business insights to become market leaders. Wamba et al. (2015) defined big data analytics as a holistic approach to capturing, managing, processing, and analyzing data.

\subsubsection{History of Big Data Analytics}

Big data analytics began with the introduction of standalone computers in businesses in the 1980s (Yaqoob et al., 2016). Leaders conducted data analytics using spreadsheets or lessadvanced technologies installed on standalone machines for the sole purpose of identifying business trends. Leaders would commonly use desktop software to perform data analytics on standalone applications with no Internet access for the sole purpose of collecting useful information for the organization. Yaqoob et al. described that by the mid-1990s, standalone applications hosted on local machines presented some challenges for leaders to accurately perform data analysis as the applications were unable to support the excessive processing loads of data for analysis.

The late 1990s saw an exponential increase in the volume of data generated globally (Balachandran \& Prasad, 2017). The volume of data increased rapidly due to the globalization of the world's economy, the emergence and ubiquity of the 
Internet, social media networks, and new product introduction of mobile devices (Amankwah-Amoah, 2016). The telecommunication sector experienced and contributed to the increase in big data mining, as the industry experienced a shift from analog to a digital platform (Asamoah et al., 2017). Data mining grew exponentially, from 281 petabytes in 1986 to 471 petabytes in 1993 , to 2.2 exabytes in 2000 , to 65 exabytes in 2007, and finally, 667 exabytes in 2010 (Hilbert \& Lopez, 2011).

The first adopters of big data analytics were startups and online organizations such as Facebook, eBay, Google, Target, Amazon, and LinkedIn; these organizations made big data analytics part of their organizations' core foundation and strategy (Ertemel, 2015). At Target, leaders used big data analytics with their loyalty card program data to track customers' buying patterns and to predict what consumers would probably buy in the future (Wamba et al., 2017). At Amazon, almost $35 \%$ of goods purchased by consumers on Amazon.com were a result of personalized recommendations targeted at each consumer (Wills, 2014).

In summary, big data analytics was introduced as a process to assist leaders with a solution to resolve the problem associated with collecting, processing, and analyzing vast amounts of data. Big data analytics provides strategic business value that offers insights to identifying trends and algorithms to predict outcomes. Big data analytics can potentially generate business value for organizations, and how and what business value is still a work in progress. The creation of big data analytics began with the introduction of numerous new tools and techniques to replace the existing ones so that leaders achieved similar or better business outcomes. With the everincreasing use of social media in the United States, a strong case exists to understand how massive amounts of collected data add business value, like increased productivity and profitability. Organizations would need to understand what benefits and challenges they may encounter.

\subsubsection{Big Data Analytics and Competitive Advantage}

Many organizational leaders have shifted to data-driven decision-making due to big data analytics's advantages (Adrian et al., 2018). Two fundamental advantages of implementing big data analytics are competitive advantage and cost savings (Mohan, 2016). The term competitive advantage refers to an organization's ability to be better than its competitors or others. Organizational leaders use their competitive advantage to build more economic value than their competitors (Manzoor et al., 2019). Organizational leaders who apply big data analytics within their organization can create a sustainable competitive advantage (Matthias et al., 2017). Leaders who invest in big data analytics have a $36 \%$ chance of improving their superiority over their competitors in areas such as operating efficiency and revenue growth (Anthony et al., 2015). Leaders use big data to leverage information in their organizations, giving them access to achieve a competitive advantage against competitors through improved performance and decision-making. For example, Walmart implemented an in-house design search engine that used big data for semantic analysis called Polaris (Raguseo \& Vitari, 2018). The Polaris system relies on text analysis from big data and machine learning to produce relevant search results. After implementing the Polaris system, the number of completed purchases online at Walmart increased from $10 \%$ to $15 \%$, thus creating a sustainable competitive advantage over their rivals (Jayanand et al., 2015).
Organizations can benefit from the use of information from big data analytics to reduce costs, save time, and make business decisions or prepare better product offers (Bumblauskas et al., 2017). Big data tools and techniques can help leaders achieve cost savings through data processing and storage of vast volumes of information at a lower price than a conventional database (Mohan, 2016). Big data technologies such as Hadoop, MongoDB, and Apache Cassandra, can provide substantial cost advantages. These technology platforms all share similar capabilities focused on storing and processing vast volumes of data for advanced analytics.

Organizational leaders can leverage big data open-source platforms to create their custom big data applications for advanced data analytics (Boncea et al., 2017). Open source big data platforms can also support integration with one another to form a single solution. For example, the Hadoop platform can blend data from different sources to produce business models that the MongoDB platform will process and produce valuable business insights in real-time. Leaders can also utilize the MongoDB platform for real-time operational processing of data (Lahmer \& Zhang, 2016). MongoDB and Hadoop are big data tools that leaders can use for data partitioning and consistency. These platforms are very similar tools but with differences in processing and storing big data to provide leaders with big data analytics options.

Organizational leaders can select big data technologies based on their organizational goals and objectives to perform advanced data analytics. Before choosing a tool, a leader should evaluate each of the big data technology solutions' advantages and disadvantages prior to committing to one. The alignment of their business requirements with a big data technology solution could increase business benefits and opportunities. Leaders also gain business insights from the data they analyze because data contain connections with distinct relationships and patterns that can translate into new business insights.

\subsubsection{Benefits of Big Data Analytics}

Balachandran and Prasad (2017) identified four benefits of big data analytics: (a) faster and better decision-making, (b) new products and services, (c) product recommendations, and (d) fraud detection. Organizational leaders seek to make faster and better decision-making strategies with big data analytics (Elgendy \& Elragal, 2016). From a leader's perspective, the significance of big data analytics lies in providing information and knowledge of value before making a business decision. Big data analytics can enable faster data analysis and decision-making through advanced data visualization.

Organizational leaders using big data analytics can create new services and products (Bartosik-Purgat \& Ratajczak-Mrożek, 2018). Leaders adopting big data technologies can improve products and services, reduce organizational costs, execute innovations faster, and make better decisions (Davenport, 2014). New product features can increase a product's value, marketability, and profitability for an organization. Organizational leaders should first implement such features on a trial basis to understand the consumers' appetite in the market before conducting a full rollout. The most frequently recognized benefits from big data technologies are related to assisting leaders in introducing better services and new products.

Leaders from online businesses who adopt big data analytics can find it a valuable strategy as it provides a significant and economical way of processing a vast amount of consumer data. When consumers utilize the Internet or mobile devices, 
they provide a great deal of information to organizations, including (a) websites frequently visited, (b) types of information viewed, and (c) the types of products or services of interest (Ahsan \& Rahman, 2016). Consumers can leave traces of the information viewed when browsing online sites (Davenport, 2014). Leaders can also gain access and process this information using big data analytics to prepare personal offers to the consumer (Almeida, 2017). Using the generated information can lead to renewed sales, improved customer services, market intelligence, and target marketing (Raguseo, 2018).

In summary, organizational leaders use big data analytics to help direct their strategy to maximize profits, reduce costs, introduce a new product, minimize fraud, and support their decision-making processes. Big data technologies allow business leaders to make proactive, knowledge-driven decisions on future trends. Nevertheless, despite the benefits and opportunities associated with big data analytics, the implementation can create challenges.

\subsubsection{Challenges to Implementing Big Data Analytics}

Big data analytics can yield beneficial information, but some challenges exist, such as (a) managing large sets of data, (b) data silos, (c) data centralization, (d) data protection and privacy, (e) management training, (f) having the right skills to conduct analysis such as data scientists, (g) cost of implementation, and (h) organizational cultural change (Saldžiūnas \& Skyrius, 2017). Michael and Miller (2013) argued that leaders would face challenges using big data analytics when trying to understand:
1. How much data to collect and store?
2. How long should the data be maintained?
3. Whether the data would be secure.
4. What is the cost?

\subsubsection{Managing large datasets}

As the volume of data increases, challenges may arise, with leaders discovering ways to manage the continued growth in data collected to yield more benefits (Saldžiūnas \& Skyrius, 2017). For example, law enforcement agencies and other organizations rely on video data generated from closed-circuit television (CCTV) from surveillance used in criminal investigations (Michael \& Miller, 2013). Leaders are challenged with deciding whether it is cost-effective to allow the closed-circuit television to run continuously or to capture only selective scenes. The large amounts of video data generated are unstructured data collected, processed, and stored using big data technology.

Unstructured data are time-consuming to process, manage and can be expensive to store for analysis using big data analytics (Michael \& Miller, 2013). A mobile network operator is a telecommunications operator or a telecom carrier. In mobile network operators (MNO), leaders in the telecommunications industry have experience managing large amounts of structured and semistructured data.

\subsubsection{Data silos}

The term data silos describe the isolation or segregation of data collection (Leonard et al., 2018). The term centralized data represents a typical nonsilo environment that consists of multiple integrated systems with a complete dataset of unstructured, semistructured, and structured data (Saldžiūnas
\& Skyrius, 2017). The term professional silos describe the process by which barriers can exist within an organization between departments, leading to isolation amongst individuals who are supposed to be working collaboratively in the same team rather than working individually (Kitchens et al., 2018). The phenomenon can be better known as divisional rivalry, departmental politics, or turf wars. Silos generally occur when leaders fail to provide themselves and their employees with a compelling reason and purpose to work together. Dismantling silo processes or systems into integrated processes or systems can be advantageous, enhancing an organization's external and internal performance.

Data silos are a frequent occurrence in the telecommunication sector, especially in mobile network operators (MNO) (Saldžiūnas \& Skyrius, 2017). The data from a mobile network operator originates from many disparate systems and sources such as (a) device data, (b) cell site data, (c) network data, and (d) back-office data. The data are scattered in standalone systems throughout the organization, making data analysis difficult. Data not centralized within an organization can present challenges for organizational leaders to produce business insights when using big data analytics.

The downside of having a predominantly silo data environment could result in the existence of data duplication in multiple data locations (Saldžiūnas \& Skyrius, 2017). Data could be of less value without cleansing, transforming, integrating, and aggregating the data before analyzing it to discover insights (Saggi \& Jain, 2018). Having a silo data environment could lead to data inaccuracy, duplicate data, and no single source of truth for an organization. A data environment that encompasses different data sources from the whole organization is complete and most valuable. Data silo environments are also not sufficiently reliable for making organizational decisions because of possible errors, duplications, and missing data.

While many organizations recognize that data silos are a problem, undoing the problem can be challenging because of the possible legacy systems that may exist or an entrenched organizational culture of separating data. Changing the mindset of employees would require organizational leaders to adopt a data centralization and sharing strategy approach.

\subsubsection{Data centralization}

In MNO organizations, billing systems are in a central location where leaders can securely access the information to conduct analytics centrally (Saldžiūnas \& Skyrius, 2017). Other systems, such as device and cell tower site information, can be located in separate systems elsewhere throughout the organization, making data analysis of the entire data challenging for leaders to derive a comprehensive view of their organization. When an organization has a centrally located data environment, it can benefit from cost savings because of its uniform data structure standards, a centrally located data environment with no duplicate data. The linking of data silos for intergenerational research can benefit from time and cost (Leonard et al., 2018). Leaders could make better decisions for their organization if the data collected were centrally hosted, structured, and easily accessible to conduct big data analytics. Despite the organizational benefit of a centrally hosted data environment to support leaders in making better decisions, consumers have concerns regarding the lack of sufficient regulatory legislation, adequate security protocols to protect and prevent a data breach on consumers' personal information. A data centralization strategy can avoid 
duplicating data sets and provide new perspectives from multiple analyses of the same data set.

\subsubsection{Data protection and data privacy}

Data protection and data privacy measures promote and justify the laws and regulations set by the regulators. Data protection and data privacy of any data collected by an organization create a concern for consumers, and it can also generate challenges for organizational leaders to manage. In Europe, for example, legal reforms on data protection and privacy exist to protect an individual's data from being sold or misused (Vayena et al., 2018). In Lithuania, by law, MNOs organizations are legally expected to store service data for a minimum of 6 months to address any future billing-related inquiries, issues, or disputes for services provided and to provide information to the appropriate authorities (Saldžiūnas \& Skyrius, 2017). The National Data Protection Regulators of the European Union are responsible for imposing significant fines of up to $2 \%$ of the global turnover on offenders of the data protection laws (Mullock, 2012).

The term data security describes the proper handling and protection of data (Sultan et al., 2018). Before organizational leaders can adopt big data analytics, they need to understand the associated requirements in ensuring data security and data privacy are part of their overall implementation solution. For example, during the data collecting, processing, and management stages of the big data process, the data are encrypted and secured to avoid tampering or manipulation. Data encryption is also applied when the data are at rest or in transit to protect the integrity of the data. Organizational leaders who choose to invest in big data analytics should adhere to data protection laws and have security measures to protect, control, access, and monitor data usage.

The term data breach describes the unintentional or intentional penetration of a secure system using agents to access private and confidential information or data to an untrusted secure environment (Sultan et al., 2018). A data breach from an internal or an external source occurs when digital agents search and exploit erroneous or insecure security protocols by using software agents to introduce malware into the intended systems (Kude et al., 2017). A data breach is an act of compromising an organization's security to gain unauthorized access to its systems and protected data (Shamsi \& Khojaye, 2018). Carphone Warehouse, a telecommunications dealer in the UK, had over 90,000 credit and debit card information of their customers stolen (Bush, 2016). The outcome of a data breach can be expensive and exorbitant for an organization because the regulatory bodies impose a hefty financial penalty and demand payouts to the parties impacted.

\subsubsection{Management training}

Another challenge of implementing big data analytics is that leaders need to acquire the analytical capability to analyze the information collected using big data analytical tools properly. Interpreting the information from big data analytical tools can be challenging if leaders do not have the required training or skills (Sharma et al., 2014). Leaders should be able to describe the output produced by using big data tools to identify strategic or operational patterns and make the right decisions to create value. Leaders derive new business insights from using big data analytical tools. When a leader cannot translate the data collected in this manner, the information produced often has little or no value to the organization (Saldžiūnas \& Skyrius, 2017). A common mistake some leaders make when using big data analytics is to focus mainly on data collection and transformation instead of using the data to develop business models and insights that might create a competitive advantage (Alharthi et al., 2017). Therefore, leaders should undergo the necessary training before proceeding with implementing big data analytics within the organization. Leaders should hire a data scientist to assist with the implementation and operational use of big data analytics technology.

Brynjolfsson et al. (2011) conducted a study that found that organizations that managed with big data increased output and productivity levels by $6 \%$, compared to other organizations that invested in only traditional internal and external data collection with fundamental analysis. The result of the study revealed that several growing organizations implemented a data-driven culture. Tambe (2014) also discovered that organizational leaders who use big data could benefit from up to $3 \%$ growth in productivity and performance than organizational leaders that do not use it. Organizations that possess employees with the knowledge and technical skills to translate the data into business insights have a competitive advantage over their rivals. It would appear that analytical skills, a driven-data culture, and proficient leaders are necessary ingredients for a successful organization. In such organizations, opportunities exist for leaders to learn new skills and for employees to harness their potential benefits of big data analytics for the sole purpose of increasing efficiency and effectiveness with the organization. Training and workplace learning efforts are also seen as the objective goals of the organization.

\subsubsection{Cost of implementation}

Economic challenges exist with implementing big data analytics. Significant capital is allocated when purchasing the hardware and software required for big data acquisition (Sivarajah et al., 2017). One of the biggest challenges with implementing big data analytics is the high cost of infrastructure (Kottasová, 2018). Even with the introduction of cloud computing technologies and other equipment, they are still costly. For example, 1,000 processing nodes connected over the cloud would require approximately 750 days to process one million gigabytes using this system and cost more than six million dollars to assemble (Trelles et al., 2011).

The costs to implement big data technology can also include hardware, software, resource time, support maintenance, and training for the employees within the organization (Verma \& Bhattacharyya, 2017). Implementations also involve setting up a robust security architecture and a governing body to monitor regulatory policies and data privacy violations internally. All these requirements and more can incur a high cost to the organizations that intend to implement and invest in big data analytics (Kottasová, 2018). It is also vital to note that the high costs in implementing big data could lead to a lower adoption rate of the technology, especially by organizational leaders in small businesses (Sharma et al., 2014).

Implementing big data analytics might be time-consuming and expensive. Still, the advantages, such as faster and better decision-making, including new products and services, could significantly outweigh the challenges or barriers over time. A survey conducted by MIT across thirty industries in over a hundred countries revealed that more high-performance organizations were using big data analytics, whereas a significant amount of low-performing organizations were not (LaValle et al., 2011). The benefits of big data analytics in terms of organizational performance are clear. The superiority 
in organization performance would be transparent within these companies. However, the investment to implement it within any company can become costly (Braganza et al., 2017).

\subsection{The Kotter's Change Model}

Kotter's change model outlines fundamental steps to implementing change. Organizational leaders can choose to use Kotter's change process model to understand the possible limitations of initiating or implementing new programs (Kotter, 2007). The model continues to be a popular source for research in change management, providing researchers with an understanding of challenges and opportunities by applying each step of the model to a specific organizational need. Kotter argued that successful implementation of change requires a series of eight phases:

1. Establish a sense of urgency regarding the need for change.

2. Build a powerful coalition.

3. Create a vision and strategy.

4. Communicate the vision.

5. Empower employees to act on the change vision.

6. Plan and create short-term wins.

7. Consolidate improvements and produce more changes.

8. Institutionalize new approaches in the corporate culture.

If used effectively, Kotter's theory can help a leader overcome opposition to change within the company and thereby promote organizational transformation and adoption (Kotter, 2007). Kotter's model provides a potential lens for understanding the change management process that the participating leaders used to implement big data analytics.

\subsubsection{Establishing a sense of urgency}

The first step of this approach was for organizational leaders to establish a sense of urgency around implementing the new systems. The type of leader and the support they have for the proposed change was crucial to the success of the initiative. Transformational leaders are more likely to motivate their workers to produce requirements for new systems and processes (Muchiri et al., 2019). Change has a better chance of succeeding through a progressive leader who can inspire at different levels within the organization (Kotter \& Schlesinger, 2008). The leader creates a sense of awareness for the organizational change to gain cooperation from the employees and their contribution to the overall outcome (Pollack \& Pollack, 2015). There is a sense of urgency and necessity to communicate the change to all employees throughout the organization. A failure to create this sense of urgency could result in employees being resistant to change and becoming defensive (Kotter, 2007). Failure in implementing change can occur when leaders do not communicate their objectives with their leaders and employees at the beginning of a business transformation. Therefore, during the change process, effective communication helps to diminish resistance (Akan et al., 2016), which could otherwise hamper the successful implementation of new policies (Nilsen et al., 2016).

\subsubsection{Build a powerful coalition}

Identifying the team to assist with implementing the change is Kotter's second stage. The second stage involves having a coalition team to implement this transformation change strategy (Kotter, 2007). Transformational change within an organization can begin with a few individuals and a dynamic leader and this is essential to creating a powerful coalition for transformational change. The team to participate in the change transformation includes members of the senior leadership, managers, and employees that are committed to the cause of improving the performance and financial stability of the organization. However, for change to succeed, the team would have the experience, commitment, credibility, knowledge, and skills to influence and mobilize change within the organization.

The coalition size is also reliant on the headcount of the organization. Therefore, the larger the size of the coalition team, the higher the chances of success. When implementing change within an organization, coalition support is essential to ensure employees' good behavior, awareness, commitment, and cooperation (Johannsdottir et al., 2015). With a great leadership team, the coalition would likely be powerful enough to implement change. Organizational leaders that do not succeed in this stage are not able to form a coalition (Kotter, 2007). Implementing a change process requires the participation and contribution of senior leaders and teams to prepare and lead the employees through the change process.

\subsubsection{Creating an organizational vision}

The third stage includes the creation of an organizational vision and strategy (Kotter, 2007). An organization's vision represents the future strategic path (Grobler et al., 2019). The vision for an organization typically comes from the initial leader of change and can extend beyond a 5-year plan. The coalition must have the same vision, strategy, and a coherent picture of the future for the organization to succeed. A realistic vision can help organizational leaders and employees align their objectives and goals. The leader of change can consider the vision as a fundamental component to motivate and inspire fellow leaders and employees within the organization. The vision contributes to the guiding principles of decision-making for the change leader while building a clear statement of direction (Pollack \& Pollack, 2015). The vision should be realistic, adaptable, communicated, focused, and achievable. Overcomplicating the vision can lead to employees being less inspired.

\subsubsection{Communicate the vision}

The fourth stage involves the communication of the vision. The change leader and coalition can sometimes fail to communicate their vision, thereby making the change process difficult for all employees to understand (Kotter, 2007). The fourth stage highlights the need for a transformation leader to effectively communicate the vision with both actions and words. Underestimating the value of creating a communication strategy to convey the change could hurt the change process (Pollack \& Pollack, 2015).

Little or no communication of the vision can also have a detrimental effect on motivating employees creating confusion and unclarity. With proper communication of the vision to all, a positive chain reaction from the employees exists to the new change process. Creating avenues for employees to discuss and provide feedback is the best way to ensure that the vision is understood and communicated (Kotter \& Schlesinger, 2008). Sharing the vision throughout the organization is an ingredient of a communication strategy for change management (Kotter, 2007). Positive and effective communication strategy for change management would remove any obstacles. 


\subsubsection{Empower employees to act on the change vision}

In the fifth stage, Kotter described the consequences of obstacles that hinder the change process' new vision. For example, the organization's size could be an obstacle to implementing change (Baloh et al., 2018). However, organizational leaders are to remove more significant obstacles for effective implementation of the change process. Other examples of change obstacles include cultural change, performance appraisal systems, and lack of employee willingness to adapt to change (Kotter, 2007). It can be challenging to encourage the support and participation of employees during the change process (Grobler et al., 2019). Some employees might be more interested in compensation than the vision (Kotter \& Schlesinger, 2008). When eliminating obstacles, it is necessary to build more integrated work processes and empower employees at different levels of the organization.

\subsubsection{Plan and create short-term wins}

Implementing a change process demands patience from the coalition (Grobler et al., 2019). The sixth step involves preparing to accept short-term wins (Kotter, 2007). The organizational willingness for change requires the engagement of leaders, managers, employees, new processes, and technology. It would take a significant amount of time for an organizational leader to achieve transformation. However, progress is something that stakeholders demand to see. Quick wins provide credibility to the change process and reassurance to the stakeholders. Leaders can bolster motivation to promote the team's focus on short-term gains. Short-term wins increase the teams' confidence, reduce complacency, and encourage detailed analytical thinking in implementing the vision and change process (Kotter \& Schlesinger, 2008).

\subsubsection{Consolidate improvements and produce more changes}

Kotter's seventh step change process cautions against premature celebrations. Implementing change can take a toll on company costs and employee morale (Kotter \& Schlesinger, 2008). Sometimes the transformation adoption can take 3 to 10 years before yielding the desired results (Kotter, 2007). Consequently, leaders must continuously sustain the drive and momentum of their employees (Grobler et al., 2019). Throughout this period, the culture within the organization was also likely to change. Organizational leaders must review the impact of the change process on the organizational culture (Willis et al., 2016a). The success of the change process requires the leadership team and employees to share the same culture, such as beliefs, assumptions, and values. Having the same culture means that the leadership team and employees solve problems and manage the risks (Mouhamadou et al., 2017). A sense of belonging and unity across all teams was also an indicator of a thriving organizational culture.

\subsubsection{Institutionalize new approaches in the corporate culture}

The eighth and final step of Kotter's change process describes the conceptual model through which organizations can regulate the change process (Kotter, 2007). When undergoing a corporate change process, employees can develop a new behavior and strategy. The employees' new behaviors become the organization's new shared values and culture. This culture can then lead to positive transformation when using Kotter's model. Transformation occurs at different organizational levels and managerial behavioral change can also occur variably in most change initiatives.

The coalition should ensure that the management and employee accept and buy into the change initiative. The change initiative should also last the duration to achieve the expected outcome and increase employee confidence. Organizational success and work behaviors can be byproducts of implementing a successful change process. Before implementing a new solution, such as big data analytics, the leader should have leadership buy-in. The leader should communicate the vision for a successful implementation throughout the organization. Members of the organization should form a coalition made up of leaders, managers, and employees who support and participate in the change process. When leaders are involved in the change process, a high probability exists that the implementation of the change, such as big data analytics, could be successful. The application of Kotter's model in this context explores strategies for successfully implementing big data, which requires a tremendous level of support from all management levels throughout the organization.

When implementing big data analytics, organizational leaders can introduce a change process that could lead to a cultural change, process improvements, and technical readiness within the organization. When implementing big data analytics, Kotter's change model can also allow for cultural alignment and effective communication within the organization. I can use Kotter's change process to explore strategies used to successfully implement big data analytics within the organizations in my study. The change process of implementing new systems requires a purposeful and successful leadership that can manage the changes related to both processes and people effectively. Using Kotter's change model, management can produce precise outcomes based on their experience, and leaders produce transformational change within the organization. Therefore, leaders and managers are vital to implementing change such as big data analytics.

\subsection{Six Sigma Model}

Six Sigma is an approach to continuous improvement, improving customer satisfaction, encouraging leadership, raising profits, and competitive advantage over other businesses (Mouaky et al., 2018). Six Sigma is also a business theory focused on continuous improvement by understanding customers' needs by analyzing business processes and establishing proper measurement methods (Samman \& Ouenniche, 2016). The Six Sigma method is a quality improvement strategy that attempts to improve efficiency (Basta et al., 2016).

Six Sigma uses two methodologies, DMAIC (Define, Measure, Analyze, Improve, and Control) and DMADV (Define, Measure, Analyze, Design, and Verify), to examine and discuss different aspects of business processes (Sony \& Naik, 2019). DMAIC and DMADV make use of statistical tools and facts to look for answers to quality-related issues and concentrate on achieving financial stability (Sony \& Naik, 2019).

The DMAIC methodology includes stages such as (a) defining specific processes, (b) use metrics to track effectiveness and evaluate efficiencies, (c) analyze data and clarify goals, (d) improve change and better alignment with corporate goals, and (e) control ongoing improvement in processes (Sony \& Naik, 2019). 
The DMADV methodology includes the following processes (a) defining specific processes of a product or service, (b) measure requirements and response, (c) analyze requirements to customer goals and needs, (d) design the improvement of business processes, and (e) verify models to check customer requirements are met along with ongoing improvements (Sony \& Naik, 2019).

\section{METHODOLOGY}

A qualitative multiple case study was used to explore strategies that IT leaders in the telecommunication industry use to implement big data analytics successfully. This study's composite conceptual framework was Kotter's change theory for driving change augmented with the Six Sigma model for business process quality management design.

Kotter developed his eight-step change model in 1995 (Kotter, 2007). If used effectively, Kotter's theory can help leaders overcome opposition to change. Kotter's model provides a potential lens for understanding the change management process used by the participating leaders to implement big data analytics. Therefore, the management and the project team's fundamental objective during the execution of big data analytics within their organization was to produce beneficial change.

Six Sigma is a framework first conceptualized in the 1980s as a model for continuous business processes improvement (Samman \& Ouenniche, 2016). Bill Smith developed the Six Sigma framework. Six Sigma is a means for finding and eliminating reasons for defects or errors, reducing cycle times and operational cost, increasing productivity, attaining higher asset utilization, and surpassing customer expectations. Two Six Sigma methodologies used are DMAIC (Define, Measure, Analyze, Improve, and Control) and DMADV (Define, Measure, Analyze, Design, and Verify). DMAIC and DMADV techniques improve the business process effectively and productively. DMADV was applied to this study. The application of big data technology in an organization also enhances business operations, financial performance, and an organization's competitive advantage. Therefore, company leaders could consider implementing big data analytics as an opportunity to eliminate waste from their current process, improve business processes, and improve organizational performance.

As such, the composite framework of Kotter's change model and the Six Sigma model was expected to be an appropriate framework for exploring and understanding the strategies that IT leaders use when implementing big data analytics successfully.

\section{SUMMARY OF THE FINDINGS}

In summary, based on the research findings, participants identified four strategies they or other IT leaders would use to implement big data analytics within an organization successfully. Data resulting from the interview process were collected via semistructured interviews through video conferencing and analysis. The data collection analysis showed that the participants achieved the successful implementation of big data analytic with the strategies identified in the themes and Kotter's eight-step model. In this study, a direct and indirect alignment existed between the themes with the conceptual framework, which previous literature also supported.

Kotter's eight-step change model has specific strategies that business leaders apply when building team stability, employee involvement, continuous progress within a company (Calegari et al., 2015). According to Trinidad (2016), some organizational leaders have successfully implemented change through efficient teamwork and communication, adequate training, and employee involvement strategies during change initiatives. The Six Sigma theory strengthened the principles of change, leadership, and project management (Wiler et al., 2017). These strategies do not directly align with the themes of the study. However, the conceptual framework was consistent with the specific improvement projects of business processes needed when following the standard DMADV fivephase pattern. Overall, to have a successful implementation of big data analytics with the organization, IT leaders would need to prepare for the change by:

1. Communicating the change in vision effectively.

2. Providing training to upskill the workforce.

3. Involving employees.

4. Building solid teams.

Leaders must prepare their employees for changes and help them cope while successfully implementing the change (Kotter, 2007). From this study, all four participants revealed the importance of the four themes mentioned in the research and how the themes contributed to understanding the strategies used to implement big data analytics.

\subsection{Analysis Summary}

The implementation of big data analytics brought about communication, training, employee involvement, and teamwork, as shown in Tables 2, 3, 4, and 5. Kotter (2007) emphasized that $50 \%$ of change implementations are doomed to fail because the leaders have not prepared the employees for the change. The failure in implementing change was an issue with which most organizational leaders are grappling.

Table 1. Profile and Demographic Information IT Leaders

\begin{tabular}{lllll}
\hline & & Participants & & \\
Parameters & & $\# 2$ & $\# 3$ & \\
\hline Participant Code & P1 & P2 & P3 & P4 \\
BDA Experience & 13 years & 11years & 14 years & 10 years \\
Year as a Leader & 20 years & 18 years & 25 years & 22 years \\
Location & L1 & L1 & L2 & L2 \\
\hline
\end{tabular}

Table 1 represents the profile and organizational demographics of the four participants. Each participant was assigned an alphanumeric value. This indicated the number of years of experience the participant had in big data analytics and the number of years as an IT leader. The two organizations are also represented in alphanumeric values. To ensure the confidentiality of the participants' names and organization information was kept hidden, identification codes to denote the combination of organization and participant such as L1P1, L1P2, L2P3, and L2P4. The alphabetic letter $L$ and the numeric values 1,2 denoted the two organizations, while the letter $\mathrm{P}$ and the numeric values 1 , 2, 3, 4 denoted the four participants taking part in the research study. The combination of the two denoted the organization and participant.

Table 2. Frequency of Communication

\begin{tabular}{lcc}
\hline $\begin{array}{l}\text { Question where participants response includes the } \\
\text { word Communication }\end{array}$ & $\begin{array}{c}\text { Times } \\
\text { Discussed }\end{array}$ & $\begin{array}{c}\text { Percentage } \\
\text { Value }\end{array}$ \\
\hline Participant L1P1, Interview question 1, 3, 4, 5, & 16 & $69 \%$ \\
Participant L1P2, Interview question 1, 3, 5 & 10 & $60 \%$ \\
Participant L2P3, Interview question 1, 3, 5, 9 & 12 & $61 \%$ \\
Participant L2P4, Interview question 1, 3, 4, 5, & 13 & $62 \%$ \\
\hline
\end{tabular}


Table 2 represents the frequency with which participants mentioned the word communication during the interview. It also shows the percentages of interview questions answered where the participants mentioned the word involvement as a strategy for implementing change initiatives. Participant L1P1 used the word communication in five of the nine interview questions, including $69 \%$ of the collected responses. Participants L2P4 and L2P3 mentioned the word communication in four of the nine interview responses, $62 \%$ and $61 \%$ of the responses received. Participant L1P2 had the least mention of the word communication in only three interview questions and had $60 \%$ in the responses.

\section{Table 3. Frequency of Training}

\begin{tabular}{lcc}
\hline $\begin{array}{l}\text { Question where participants response includes the } \\
\text { word Training }\end{array}$ & $\begin{array}{c}\text { Times } \\
\text { Discussed }\end{array}$ & $\begin{array}{c}\text { Percentage } \\
\text { Value }\end{array}$ \\
\hline Participant L1P1, Interview question 1, 3, 7, 8 & 12 & $61 \%$ \\
Participant L1P2, Interview question 1, 3, 8 & 8 & $48 \%$ \\
Participant L2P3, Interview question 1, 3, 5, 8 & 13 & $62 \%$ \\
Participant L2P4, Interview question 1, 3, 5, 8 & 10 & $60 \%$ \\
\hline
\end{tabular}

Table 3 includes the frequency with which participants discussed training employees and IT leaders throughout the interview process and the percentage of interview questions answered concerning training as a strategy for implementing big data analytics change initiatives. Participant L2P3 discussed training in four of the nine interview questions, inclusive of $62 \%$ of the responses collected. It was the highest response obtained. Participants L1P1 and L2P4 also discussed training in four of the nine interview questions, which were $61 \%$ and $60 \%$ of participants' responses. Participant L1P2 referenced training in three of nine interview questions: the lowest participants' responses of $48 \%$.

Table 4. Frequency of Employee Involvement

\begin{tabular}{lcc}
\hline $\begin{array}{l}\text { Question where participants response includes the } \\
\text { word Employee Involvement }\end{array}$ & $\begin{array}{c}\text { Times } \\
\text { Discussed }\end{array}$ & $\begin{array}{c}\text { Percentage } \\
\text { Value }\end{array}$ \\
\hline Participant L1P1, Interview question 1, 3, 5, & 8 & $47 \%$ \\
Participant L1P2, Interview question 1, 3, 5, & 12 & $61 \%$ \\
Participant L2P3, Interview question 1, 3, 5, & 8 & $47 \%$ \\
Participant L2P4, Interview question 1, 3, 5, & 9 & $49 \%$ \\
\hline
\end{tabular}

As shown in Table 4, the frequency with which participants mentioned the phrase employee involvement during the interview. It also shows the percentages of interview questions answered were the participants mentioned the word involvement as a strategy for implementing change initiatives. Participant L1P2 discussed employee involvement in four of the nine interview questions, inclusive of $61 \%$ of the responses collected. Participant L2P4 discussed employee involvement in three of the nine interview responses, which was $49 \%$ of the responses received. Participant L1P1 and L2P3 referenced employee involvement three of nine interview responses, which was $47 \%$ of the responses, which constituted the lowest responses collected.

Table 5. Frequency of Teamwork

\begin{tabular}{lcc}
\hline $\begin{array}{l}\text { Question where participants response includes the } \\
\text { word Teamwork }\end{array}$ & $\begin{array}{c}\text { Times } \\
\text { Discussed }\end{array}$ & $\begin{array}{c}\text { Percentage } \\
\text { Value }\end{array}$ \\
\hline Participant L1P1, Interview question 1, 2, 3, 4, & 15 & 68 \\
Participant L1P2, Interview question 1, 3, 5, 9 & 11 & 60 \\
Participant L2P3, Interview question 1, 3, 5, 9 & 14 & 64 \\
Participant L2P4, Interview question 1, 3, 4, 5, & 11 & 60 \\
\hline
\end{tabular}

As shown in Table 5, participant L1P1 had the highest level of frequency of teamwork, with $68 \%$ of the data collected including a reference to the theme teamwork. This was followed by participant L2P3 with $64 \%$ and both participant L1P2 and L2P4 with 60\%, the lowest frequency.

\subsection{Findings}

The applicability of the findings concerning professional business practice detailed what IT leaders consider essential strategies to successfully implement a change initiative such as big data analytics within the telecommunication industry. Dynamic change strategies are necessary for organizational leaders to adapt, lead their organizations, and remain viable in a competitive environment (Yi et al., 2016). This study may help corporate leaders who attempt to implement a change initiative successfully within their organization. Based on Kotter (2007), an organizational leader's immediate need is to remain competitive and survive over time. Leaders have to understand the tools for implementing change to succeed and keep their competitive advantage in a highly volatile environment (Jeong \& Shin, 2019). The findings of this study may have a positive influence on identifying these strategies.

Overall, the qualitative results are relevant to IT leaders because they provide a more profound understanding of the essential elements linked to implementing big data analytics successfully. By analyzing the IT leaders' expectations and experiences, we could see crucial strategies for designing and implementing a successful big data analytics project from using a multiple case study design. The findings also indicated that the strategies were critical to the organization's project team adoption and delivery of big data projects successfully within their respective organizations. The participants emphasized the significance of being proactive, having open and continuous communication, providing adequate training and workshops, defining clear roles and responsibilities, having a top-down, bottom-up approach with employee involvement, and creating a thriving teamwork culture. The participants also insisted on the importance of having a working process model and documenting the entire processes across the different functional areas, and reusing the same successful model again and again for future implementations. IT leaders in the telecommunication industry must understand how to successfully implement big data analytics because of the benefits it brings to the organization to remain competitive. This study's significance would help determine the best strategies and techniques for IT leaders to use to implement big data analytics.

Theoretically, it appears simple to note the critical success indicators for implementing big data analytics projects, but reality showed that there was still a high project failure rate. More complicated sets of problems or profound project implications could influence the project failure rate, but that will need further investigation. This study highlighted the relationship between management and employees and some tangible strategies used during the implementation process. These strategies serve as a foundation for a standardized change initiative process for IT leaders to reduce high failure rates. The findings of this study could also serve as a foundation to increase productivity and minimize financial losses. IT leaders who successfully implement change initiatives can increase productivity, reduce financial losses, and become more competitive. All four themes that emerged from the study aligned with Kotter's eight-step change model but only partially aligned with the Six Sigma methodology DMADV.

The four themes of (a) communication, (b) training, (c) employee involvement in decisions, and (e) teamwork correlated and aligned with several of Kotter's model steps, such as (a) establish a sense of urgency regarding the need for change, (b) build a powerful coalition, (c) create a vision and strategy, (d) communicate the vision, (e) empower employees 
to act on the change vision, (f) plan and create for short-term wins, are essential and necessary to ensure a successful implementation of a change initiative. DMADV methodology develops the ideal business model destined to satisfy the customers' needs. The method is associated with the creation of new services and products. The crucial thing to note is that the product or service did not exist. It is also essential to know that the DMADV methodology could be used for new and existing processes to improve performance and technology, but it sometimes provides unfortunate outcomes. This study focused on introducing new technology into the organization.

Nevertheless, the DMADV methodology supports identifying areas for improvement when developing a new product or process. The study's result-focused on identifying strategies for successfully implementing big data analytics and not identifying areas for improvement. The DMADV methodology does not show a direct correlation to the themes identified in the study. It was also important to note that none of the participants had mentioned they used any conceptual framework or theories during the implementation of big data analytics projects.

Kotter's eight-step change and Six Sigma change models provided the conceptual framework for this research. The four themes were directly aligned to Kotter's change models and partially to Six Sigma. The data analysis revealed that IT leaders used communication, training, and employees' involvement and teamwork to implement big data analytics in their respective organizations successfully. According to Babbie (2015), IT leaders could successfully recognize strategies that other IT leaders have used to implement big data analytics successfully. Though the participants had a limited perspective of the conceptual framework, they achieved success when implementing big data analytics.

\section{CONCLUSION}

The purpose of this qualitative multiple case study was to identify strategies that IT leaders use to implement big data analytics successfully. Although many big data analytics implementation trials fail, the results of the study may provide IT leaders with some strategies to successfully implement big data analytics. Change remains inevitable as organizations try to survive in the ever-changing economy. Therefore, implementing change initiatives was crucial to organizational success. Though 22 themes emerged from the analysis, only four themes were considered effective strategies. All four participants attained success in their projects and suggested the necessity of effective communication between management and employees, training and workshop sessions to all involved, employee involvement earlier in the change process, and efficient and effective teamwork with the project teams.

Overall, the qualitative results are of relevance to IT leaders because they provide a more profound understanding of the essential elements linked to implementing big data analytics successfully. By analyzing the IT leaders' expectations and experiences, we could see crucial strategies used for the design and implementation of a successful big data analytics project from using a multiple case study design.

The results from this study showed that although the failure to implement big data analytics was considerable, using the suggested strategies identified could increase the success of implementing this change initiative. Therefore, the results did provide IT leaders in the telecommunication industry with valuable insight on how to use strategies when implementing a change initiative or to implement big data analytics successfully in the future.

\section{REFERENCES}

[1] Grover, V., Chiang, R. H. L., Liang, T. P., \& Zhang, D. (2018). Creating strategic business value from big data analytics: A research framework. Journal of Management Information Systems, 35, 388-423. https://doi.org/10.1080/07421222.2018.1451951

[2] Chrimes, D., Moa, B., Kuo, M. A., \& Kushniruk, A. (2017). Operational efficiencies and simulated performance of big data analytics platform over billions of patient records of a hospital system. Advances in Science, Technology and Engineering Systems, 2(1), 2341. https://doi.org/10.25046/aj020104

[3] Matthias, O., Fairweather, I., Gregory, I., \& Vernon, A. (2017). Making sense of big data can it transforms operations management? International Journal of Operations \& Production Management, 37(1), 37-55. https://doi.org/10.1108/IJOPM-02-2015-0084

[4] Alsghaier, H., Akour, M., Shehabat, I., \& Aldiabat, S. (2017). The importance of big data analytics in business: A case study. American Journal of Software Engineering and Applications, 6(4), 111-115. https://doi.org/10.11648/j.ajsea.20170604.12

[5] Sharma, R., Mithas, S., \& Kankanhalli, A. (2014). Transforming decision-making processes: A research agenda for understanding the impact of business analytics on organizations. European Journal of Information Systems, 23, 433-441. https://doi.org/10.1057/ejis.2014.17

[6] Wang, Y., \& Hajli, N. (2017). Exploring the path to big data analytics success in healthcare. Journal of Business Research, https://doi.org/10.1016/j.jbusres.2016.08.002

$70,287-299$

[7] Balachandran, B. M., \& Prasad, S. (2017). Challenges and benefits of deploying big data analytics in the cloud for business intelligence. Procedia Computer Science, 112, https://doi.org/10.1016/j.procs.2017.08.138

[8] Wamba, S. F., Akter, S., Edwards, A., Chopin, G., \& Gnanzou, D. (2015). How 'big data' can make big impact: Findings from a systematic review and a longitudinal case study. International Journal of Production Economics, 165, 234-246. https://doi.org/10.1016/j.ijpe.2014.12.031

[9] Yaqoob, I., Hashem, I. A. T., Gani, A., Mokhtar, S., Ahmed, E., Anuar, N. B., \& Vasilakos, A. V. (2016). Big data: From beginning to future. International Journal of Information Management, 36, 1231-1247. https://doi.org/10.1016/j.ijinfomgt.2016.07.009

[10] Abolfazli, S., Sanaei, Z., Gani, A., Xia, F., \& Yang, L. T. (2014). Rich mobile applications: genesis, taxonomy, and open issues. Journal of Network \& Computer Applications, $\quad 40(1), \quad 345-362$. https://doi.org/10.1016/j.jnca.2013.09.009

[11] Kacprzyk, J., \& Zadrożny, S. (2001). Computing with words in intelligent database querying: standalone and internet-based applications. Information Sciences, 134(1), 71-109. https://doi.org/10.1016/S00200255(01)00093-7 
[12] Adnan, K., \& Akbar, R. (2019). An analytical study of information extraction from unstructured and multidimensional big data. Journal of Big Data, 1, 1-38. https://doi.org/10.1186/s40537-019-0254-8

[13] Woo, H., Kim, K., Cha, K., Lee, J. Y., Mun, H., Cho, S. J., \& Kang, M. (2019). Application of efficient data cleaning using text clustering for semistructured medical reports to large-scale stool examination reports: Methodology study. Journal of Medical Internet Research, 21(1), 1-6. https://doi.org/10.2196/10013

[14] Nassiri, H., Machkour, M., \& Hachimi, M. (2018). One query to retrieve $\mathrm{xml}$ and relational data. Procedia Computer Science, 134, 340-345. https://doi.org/10.1016/j.procs.2018.07.201

[15] Wu, P. J., \& Lin, K. C. (2018). Unstructured big data analytics for retrieving e-commerce logistics knowledge. Telematics and Informatics, 35(1), 237-244. https://doi.org/10.1016/j.tele.2017.11.004

[16] Manzoor, A., Khan, N. R., \& Adeel, K. (2019). An empirical analysis of the buyer's perspective of gaining competitive advantage through supplier development. South Asian Journal of Management Sciences, $\quad 13(1), \quad 56-73$. https://doi.org/10.21621/sajms.2019131.04

[17] Anthony, M., Stefan, M., \& Rebecca, S. (2015). How leading organizations use big data and analytics to innovate. Strategy \& Leadership, 43(5), 32-39. https://doi.org/org/10.1108/SL-06-2015-0054

[18] Raguseo, E., \& Vitari, C. (2018). Investments in big data analytics and firm performance: An empirical investigation of direct and mediating effects. International Journal of Production Research, 56, 5206-5221. https://doi.org/10.1080/00207543.2018.1427900

[19] Jayanand, M., Kumar, M. A., Srinivasa, K. G., \& Siddesh, G. M. (2015). Big data computing strategies. Handbook of research on securing cloudbased databases with biometric applications, 72(1), 7291. $\quad$ https://doi.org/org/10.4018/978-1-4666-6559$0 . \operatorname{ch} 004$

[20] Bumblauskas, D., Nold, H., Bumblauskas, P., \& Igou, A. (2017). Big data analytics: Transforming data to action. Business Process Management Journal, 23, 703720. https://doi.org/10.1108/BPMJ-03-2016-00.6

[21] Mohan, A. (2016). Big data analytics: Recent achievements and new challenges. International Journal of Computer Applications Technology and Research 5, 460-464. https://doi.org/10.7753/ijcatr0507.1008

[22] Boncea, R., Petre, I., Smada, D. M., \& Zamfiriroiu, A. (2017). A maturity analysis of big data technologies. Informatica Economica, 21(1), 60-71. https://doi.org/10.12948/issn14531305/21.1.2017.05

[23] Lahmer, I., \& Zhang, N. (2016). Towards a virtual domain based authentication on map reduce. IEEE Access, 4, 1658-1675. https://doi.org/10.1109/ACCESS.2016.2558456

[24] Elgendy, N., \& Elragal, A. (2016). Big data analytics in support of the decision making process. Procedia Computer Science, 100, 1071-1084. https://doi.org/10.1016/j.procs.2016.09.251
[25] Bartosik-Purgat, M., \& Ratajczak-Mrożek, M. (2018). Big Data Analysis as a Source of Companies' Competitive Advantage: A Review. Entrepreneurial Business \& Economics Review, 6(4), 197-215. https://doi.org/10.15678/EBER.2018.060411

[26] Davenport, T. H. (2014).Big Data @ Work. Harvard Business School. https://doi.org/10.15358/9783800648153

[27] Ahsan, K., \& Rahman, S. (2016). An investigation into critical service determinants of customer to business (C2B) type product returns in retail firms. International Journal of Physical Distribution \& Logistics Management, 46, 606-633. https://doi.org/10.1108/JJPDLM-09-2015-0235

[28] Almeida, F. L. F. (2017). Benefits, challenges and tools of big data management. Journal of Systems Integration (1804-2724), $\quad 8(4), \quad 12-20$ https://doi.org/org/10.20470/jsi.v8i4.311

[29] Raguseo, E. (2018). Big data technologies: An empirical investigation on their adoption, benefits and risks for companies. International Journal of Information Management, 38(1), https://doi.org/10.1016/j.ijinfomgt.2017.07.008

[30] Saldžiūnas, K., \& Skyrius, R. (2017). The challenges of big data analytics in the mobile communications sector. Ekonomika / Economics, 96(2), 110-121. https://doi.org/10.15388/Ekon.2017.2.11004

[31] Michael, K., \& Miller, K. W. (2013). Big Data: New opportunities and new challenges. Computer, 46(6), 2224. https://doi.org/10.1109/MC.2013.196

[32] Leonard, D., Buettner, P., Thompson, F., Makrides, M., \& McDermott, R. (2018). Linking "data silos" to investigate anaemia among aboriginal and torres strait islander mothers and children in far North Queensland." Australian and New Zealand Journal of Public Health, 42, 456-462. https://doi.org/10.1111/1753-6405.12821

[33] Kitchens, B., Dobolyi, D., Jingjing, L. I., \& Abbasi, A. (2018). Advanced customer analytics: Strategic value through integration of relationship-oriented big data. Journal of Management Information Systems, 35 , 540-574. https://doi.org/10.1080/07421222.2018.1451957

[34] Saggi, M. K., \& Jain, S. (2018). A survey towards an integration of big data analytics to big insights for valuecreation. Information Processing \& Management, 54(5), 758-790. https://doi.org/10.1016/j.ipm.2018.01.010

[35] Vayena, E., Dzenowagis, J., Brownstein, J. S., \& Sheikh, A. (2018). Policy implications of big data in the health sector. Bulletin of the World Health Organization, 66-68. https://doi.org/10.2471/BLT.17.197426

[36] Mullock, J. (2012). EU data privacy laws face big shakeup. Journal of Direct, Data \& Digital Marketing Practice, 13, 369-371. https://doi.org/10.1057/dddmp.2012.6

[37] Sultan, K., Ali, H., \& Zhang, Z. (2018). Big data perspective and challenges in next generation Networks. Future Internet, 10(7), 1-20 https://doi.org/10.3390/fi10070056 
[38] Kude, T., Hoehle, H., \& Sykes, T. A. (2017). Big data breaches and customer compensation strategies Personality traits and social influence as antecedents of perceived compensation. International Journal of Operations \& Production Management, 37(1), 56-74. https://doi.org/10.1108/IJOPM-03-2015-0156

[39] Shamsi, J. A., \& Khojaye, M. A. (2018). Understanding privacy violations in big data systems. IT Professional, 20(3), 73-81. https://doi.org/10.1109/MITP.2018.032501750

[40] Bush, D. (2016). How data breaches lead to fraud. Network Security, 2016(7), 11-13. https://doi.org/10.1016/S1353-4858(16)30069-1

[41] Alharthi, A., Krotov, V., \& Bowman, M. (2017). Addressing barriers to big data. Business Horizons, 60(3), 285-292. https://doi.org/10.1016/j.bushor.2017.01.002

[42] Brynjolfsson, E., Hitt, L. M., \& Kim, H. H. (2011) Strength in numbers: how does data-driven decision making effect firm performance? https://doi.org/10.2139/ssrn.1819486

[43] Tambe, P. (2014). "Big data investment, skills, and firm value." Management $\quad$ Science, 60, 1452-1469. https://doi.org/10.1287/mnsc.2014.1899

[44] Sivarajah, U., Kamal, M. M., Irani, Z., \& Weerakkody, V. (2017). Critical analysis of Big Data challenges and analytical methods. Journal of Business Research, 70, 263-286. https://doi.org/10.1016/j.jbusres.2016.08.001

[45] Trelles, O., Prins, P., Snir, M., \& Jansen, R. C. (2011). Big data, but are we ready? Nature Reviews. Genetics, 12, 224. https://doi.org/10.1038/nrg2857-c1

[46] Verma, S., \& Bhattacharyya, S. S. (2017). Perceived strategic value-based adoption of big data analytics in emerging economy. A qualitative approach for Indian firms. Journal of Enterprise Information Management, 30, 354-382. https://doi.org/10.1108/JEIM-10-2015-0099

[47] LaValle, S., Lesser, E., Shockley, R., Hopkins, M. S., \& Kruschwitz, N. (2011). Big data, analytics, and the path from insights to value. MIT Sloan Management Review, 52(2), 21-32. Sloan Management Review. https://sloanreview.mit.edu

[48] Braganza, A., Brooks, L., Nepelski, D., Ali, M., \& Moro, R. (2017). Resource management in big data initiatives: Processes and dynamic capabilities. Journal of Business Research, 70, 328-337. https://doi.org/10.1016/j.jbusres.2016.08.006

[49] Kotter, J. P. (2007). Leading change: Why transformation efforts fail. Harvard Business Review, 85(1), 96-103. Harvard Business Review. https://hbr.org

[50] Muchiri, M. K., McMurray, A. J., Nkhoma, M., \& Pham, H. C. (2019). How transformational and empowering leader behaviors enhance workplace safety: A review and research agenda. Journal of Developing Areas, 53(1), 257-265. https://doi.org/10.1353/jda.2019.0015

[51] Kotter, J. P., \& Schlesinger, L. A. (2008). Choosing strategies for change. Harvard Business Review, 86(8), 128-130. Harvard Business Review. https://hbr.org
[52] Pollack, J., \& Pollack, R. (2015). Using Kotter`s eight stage process to manage an organizational culture change program: Presentation and practice. Systematic Practice and Action Research, 28, 51-66. https://doi.org/10.1007/s11213-014-9317-0

[53] Johannsdottir, L., Olafsson, S., \& Davidsdottir, B. (2015). Leadership role and employee acceptance of change. Journal of Organizational Change Management, 28(1), 72-96. https://doi.org/10.1108/JOCM-12-2013-0238

[54] Grobler, C., Wyk, R. V., \& Magau, M. D. (2019). Transformational change leadership framework for implementing broad-based black economic empowerment in south african organizations. Acta Commercii 19(1), 1-12 https://doi.org/10.4102/ac.v19i1.686

[55] Baloh, J., Zhu, X., \& Ward, M. M. (2018). Implementing team huddles in small rural hospitals: How does the Kotter model of change apply? Journal of Nursing Management, $\quad 26, \quad 571-578$ https://doi.org/10.1111/jonm.12584

[56] Willis, C. D., Saul, J., Bevan, H., Scheirer, M. A., Best, A., Greenhalgh, T., \& ... Bitz, J. (2016a). Sustaining organizational culture change in health systems. Journal of Health Organization and Management, 30(1), 2-30. https://doi.org/10.1108/JHOM-07-2014- 0117

[57] Mouhamadou, S., Jeanie, M., \& Rosa, O. (2017). The relationship between leadership style, organizational culture, and job satisfaction in the U.S. healthcare industry. Management and Economics Research Journal, 3, 1-10. https://doi.org/10.18639/MERJ.2017.03.403737

[58] Mouaky, M., Benabbou, L., \& Berrado, A. (2018). Dmadv approach to evaluate the adaptive kanban performance for inventory management process: The case of Moroccan public pharmaceutical supply chain. Supply Chain Forum: International Journal, 19(3), 178-190. https://doi.org/10.1080/16258312.2018.1484249

[59] Samman, R. A., \& Ouenniche, J. (2016). Continuous quality improvement programs - part I: survey, critical analysis and future research directions. Journal of Developing Areas, 50(4), 39-66. https://doi.org/10.1353/jda.2016.0152

[60] Calegari, M. F., Sibley, R. E., \& Turner, M. E. (2015). A roadmap for using Kotter's organizational change model to build faculty engagement in accreditation. Academy of Educational Leadership Journal, 19(3), 31-43. https://www.alliedacademies.org

[61] Wiler, J. L., Bookman, K., Birznieks, D. B., Leeret, R., Koehler, A., Planck, S., \& Zane, R. (2017). Rapid process optimization: A novel process improvement methodology to innovate health care delivery. American Journal of Medical Quality, 32, 172-177. https://doi.org/10.1177/1062860616637683

[62] Yi, Y., Li, Y., Hitt, M. A., Liu, Y., \& Wei, Z. (2016). The influence of resource bundling on the speed of strategic change: Moderating effects of relational capital. Asia Pacific Journal of Management, 33, 435467. https://doi.org/10.1007/s10490-016-9458-z

[63] Jeong, I., \& Shin, S. J. (2019). High-performance work practices and organizational creativity during 
International Journal of Computer Applications Technology and Research

Volume 10-Issue 04, 64-76, 2021, ISSN:-2319-8656

organizational change: a collective learning perspective. Journal of Management, 45(3), 909-925. https://doi.org/10.1177/0149206316685156

[64] Babbie, E. (2015). Practice of social research. Cengage Learning. 